\title{
Maintenance or Emergence of Chronic Phase Secondary Cytotoxic T Lymphocyte Responses after Loss of Acute Phase Immunodominant Responses Does Not Protect SIV-Infected Rhesus Macaques from Disease Progression
}

\author{
M. Shannon Keckler, ${ }^{1,2,3}$ Vida L. Hodara, ${ }^{2,4}$ Laura M. Parodi, ${ }^{2}$ and Luis D. Giavedoni, 4 \\ ${ }^{1}$ University of Texas Health Science Center at San Antonio, 7703 Floyd Curl Drive, San Antonio, TX 78229-3900, USA \\ ${ }^{2}$ Department of Virology and Immunology, Southwest Foundation for Biomedical Research, 7620 NW Loop 410, \\ San Antonio, TX 78227-5301, USA \\ ${ }^{3}$ Poxvirus and Rabies Branch, Centers for Disease Control, 1600 Clifton Road MS-G06, Atlanta, GA 30333, USA \\ ${ }^{4}$ Southwest National Primate Research Center, Southwest Foundation for Biomedical Research, 7620 NW Loop 410, \\ San Antonio, TX 78227-5301, USA
}

Correspondence should be addressed to Luis D. Giavedoni, lgiavedoni@sfbr.org

Received 30 November 2009; Revised 19 February 2010; Accepted 9 March 2010

Academic Editor: Kim Klonowski

Copyright ( $\odot 2010$ M. Shannon Keckler et al. This is an open access article distributed under the Creative Commons Attribution License, which permits unrestricted use, distribution, and reproduction in any medium, provided the original work is properly cited.

\begin{abstract}
The simian immunodeficiency virus- (SIV-) infected rhesus macaque is the preferred animal model for vaccine development, but the correlates of protection in this model are not completely understood. In this paper, we document the cytotoxic T lymphocyte (CTL) response to SIV and its effects on viral evolution in an effort to identify events associated with disease progression regardless of MHC allele expression. We observed the evolution of epitopes targeted by CTLs in a group of macaques that included long-term nonprogressing (LTNP), slowly progressing (SP), normally progressing (NP), and rapidly progressing (RP) animals. Collectively, our data (1) identify novel CTL epitopes from an SP animal that are not restricted by known protective alleles, (2) illustrate that, in this small study, RP and NP animals accrue more mutations in CTL epitopes than in SP or LTNP macaques, and (3) demonstrate that the loss of CTL responses to immunodominant epitopes is associated with viral replication increases, which are not controlled by secondary CTL responses. These findings provide further evidence for the critical role of the primary cell-mediated immune responses in the control of retroviral infections.
\end{abstract}

\section{Background}

There is compelling evidence that the virus-specific cytotoxic $\mathrm{T}$ lymphocyte (CTL) response is an important factor in the control of human and simian immunodeficiency viruses (HIV and SIV, resp.) in infected individuals. Failures in antibody-based vaccines emphasize the importance of the CD8+ T lymphocyte response, but the disappointing results of the STEP HIV vaccine trial indicates a need for a more complete understanding of CTL responses in lentiviral infections [1]. The CTL response is restricted by the repertoire of major histocompatibility complex (MHC) Class I molecules that present viral epitopes, and a greater knowledge of these allele: epitope combinations is vital for identifying antigenspecific CTLs and measuring effector functions of antiviral cellular immunity. In addition, more information is needed about the selective pressure applied by CTLs to viral epitopes and how that influences viral evolution during SIV infection [2].

The expression of the rhesus macaque major histocompatibility complex (MHC) Class I molecule Mamu $A^{*} 01$ has been determined to be associated with slower disease progression $[3,4]$, which is similar to the protective effects of HLA $B^{*} 57$ expression in humans [5]. One important technological development from this research was the creation of Mamu $A^{*} 01$ tetramers [6]. Unfortunately, animals with 
Mamu $A^{*} 01$ are in great demand, and the limited availability of these animals has slowed research in this area [7]. Other protective alleles include Mamu $B^{*} 17$, which was initially reported as a protective allele that was enriched in longterm nonprogressor (LTNP) animals [8], but recent work indicates that animals with different $M a m u B^{*} 17$ haplotypes have divergent disease courses [9]. A third Mamu allele$B^{*} 08$ - was also reported to be associated with slower disease progression and is enriched in macaque populations that exhibit LTNP phenotypes [10].

CTL responses during HIV and SIV infection generally consist of several CTL clones recognizing many different epitopes; the size of those CTL clones can differ substantially resulting in dominant and subdominant responses [11, 12]. Immunodominance in CTL response is influenced by several factors [13], including MHC expression [14], T cell avidity $[15,16]$, proteosome processing [17], frequency of naïve precursors specific for a viral epitope [18], and viral sequence variability [19]. It has been shown that in SIV infection CTL selective pressure is a cause of viral escape mutations [20-23], and this phenomenon is one, but not the only, cause of vaccine failure $[24,25]$. Escape variants have been identified and characterized in Mamu $A^{*} 01-[26,27], B^{*} 08$ [23], and $B^{*} 17$-restricted [25] CTL epitopes, and it has been shown that these viral escape variants are poorly recognized by subsequent MHC-matched hosts but can revert to wild type if CTL selective pressure is removed by transmitting the mutated viruses to MHC mismatched animals [28-30]; interestingly, SIV can also adapt to the fitness cost of certain escape mutations by generating compensatory mutations [29].

This paper presents detailed results from a group of eight rhesus macaques followed for over 4 years (or until progression to disease) to determine the interactions between the CTL response and viral evolution in chronic SIV infection. Our comprehensive analysis of CTL response monitoring and viral sequencing in animals of varying disease progression phenotypes enabled us to identify novel epitopes recognized by antiviral CTL responses. We also show that, in this study, slow progressors (SPs) and LTNP animals accrue fewer mutations in CTL epitopes than either normal or rapid progressor animals (NP and RP, resp.), and that increased mutations are associated with a lower CD4+ T cell count. Most importantly, we demonstrate that a loss of CTL response to immunodominant epitopes can be associated with disease progression despite the presence of other subdominant CTL responses. This was observed in an animal that expressed the protective $M a m u A^{*} 01$ allele as well as an animal that expressed no known protective allele.

\section{Materials and Methods}

2.1. Experimental Animals and Tether System. Animal care and treatment were in accordance with SFBR Institutional Animal Care and Use Committee (IACUC) approved protocols. Four Indian origin rhesus macaques $(16037,16040$, 16041, and 16044), which had been vaccinated 24 months prior to this experiment with a Nef-deleted SIVmac239 live attenuated vaccine [31], were determined to be virus negative by RT-PCR and virus isolation at the time of challenge. Four additional Indian-origin, retrovirus-negative naïve rhesus macaques $(11896,12416,12900$, and 14757) were used as a control group. These eight animals were placed on a tether system during the first 4 weeks of infection in order to demonstrate the usefulness of this technology in the study of viral immunology during the acute phase of infection. Details of the placement of the tether system, which allowed for blood sampling without sedation, are provided elsewhere [32].

2.2. Viral Challenge and Viral Loads. The animals were challenged intravenously with $1 \mathrm{~mL}$ of the pathogenic isolate SIVmac251 at a dose of 100 TCID $_{50}$. PBMCs were isolated by Ficoll-Hypaque separation for use in IFN- $\gamma$ ELISPOT assays, CTL functional assays, flow cytometry, and viral isolation. Lymphocyte phenotyping was performed by multicolor flow cytometry as described elsewhere [31]. Plasma was utilized to determine viral loads by (nucleic acid sequence based analysis) NASBA assay (from Advanced Bioscience Labs, Inc.) with a lower detection limit of 500 RNA copies per $100 \mu \mathrm{L}$ plasma. NASBA technology was utilized instead of real-time RT-PCR because all the plasma samples collected while animals were on tether contained sodium heparin. For relevant comparison, the chronic viral loads were also performed with this assay.

2.3. MHC Allele Genotyping. RNA was isolated from macaque PBMCs with the RNAeasy kit (Qiagen). RNA was converted to CDNA by amplification with reverse transcriptase and random primers. This $\mathrm{cDNA}$ was then subjected to PCR with a forward primer common for the human A, B, and $\mathrm{C}$ alleles and reverse primers specific for either alleles $\mathrm{A}$ or $\mathrm{B}$ [33]. The amplified fragments were cloned into the pCR2.1 (Invitrogen) plasmid and sequenced. In addition, animals were screened for Mamu $A^{*} 01, A^{*} 02, B^{*} 08$, and $B^{*} 17$ by sequence-specific PCR as previously described in [34].

\subsection{CTL Response Measurement and Epitope Identification} by ELISPOT Assay. Peptide-specific release of IFN- $\gamma$ from lymphocytes was measured by ELISPOT in accordance with the manufacturer's instructions (U-Cytech, Utrecht, The Netherlands). SIV-specific responses were determined with 15-mer overlapping SIV peptides (NIH AIDS Research and Reference Reagent Program) at a concentration of $2 \mu \mathrm{g} / \mathrm{mL}$. All conditions were performed in duplicate with a positive control consisting of cells stimulated with Staphylococcus enterotoxin A and B (SEA/B), and a negative control of RPMI containing $0.5 \%$ dimethyl sulfoxide (DMSO) (Sigma) alone. To minimize bias, all plates were counted by one individual in a blinded fashion.

Responses were normalized for CD8+ T cells determined by flow cytometry and adjusted for DMSO background per animal. For those epitopes of known restriction, tetramer studies were performed to ensure that it was the CD8+ T lymphocytes that recognized the epitope (data not shown). For epitopes of unknown restriction, CD107 and Cytoxilux 
(OncoImmunin, Inc.) assays were utilized to determine that apoptosis of target autologous lymphoblastoid B cells was peptidespecific and that the CD8+ T lymphocyte population was positive for degranulation in a peptide-specific manner [32]. Epitopes identified by IFN- $\gamma$ ELISPOT are putative only, because they were determined by single 15-mer peptides or overlapping regions of two or more 15-mer peptides that contain the 8- to 10 -amino acid epitope. To identify these putative epitopes, PBMCs from each animal were utilized in ELISPOT assays with peptide pools representing the full SIVmac239 amino acid sequence. Positive pools were split into smaller pools, and assays were repeated. This deconvolution continued until either one single 15-mer peptide or two overlapping 15-mer peptides were identified as peptides that elicited production of IFN $\gamma$. No additional mapping of epitopes was performed in this study.

2.5. Virus Isolation and Epitope Sequencing. PBMCs from infected animals were cocultured with CEMx174 cells with or without CD8+ lymphocyte depletion with magnetic beads coated with anti-CD8+ antibodies (Dynal). Supernatant from these cultures was assayed by ELISA for SIV gag protein p27 [31]. Infected CEMx174 cells from ELISApositive samples were collected and DNA extracted with the PureGene DNA Isolation Kit (Gentra Systems) in accordance with the manufacturer's protocols. The DNA was then used in PCR reactions to amplify fragments of SIV provirus containing relevant epitopes for Gag, Rev, Vif, Tat, Pol, and Nef. The primers employed were as follows: $\mathrm{Gag}_{F}$, 5'GCCTGGTCAACTCGGTACTC3'; Gag ${ }_{R}$, 5'GTGGACCTAACTCTATTCCTGTTACAA3'; Vif $_{F}, 5^{\prime}$ GAAGGGACCCGGTGAGCTATTG3'; Vif $_{R}$, 5'AGGAGGAGGTCCTGG-

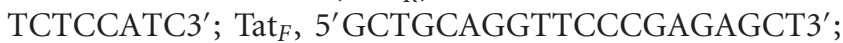
$\mathrm{Tat}_{R}, 5^{\prime}$ ACAAAACTGGCAATGGTAGCAACACT3'; $\mathrm{Pol}_{F}$, $5^{\prime}$ CAGGTCCCAAAATTCCACTTACCAG3' ${ }^{\prime}, \mathrm{Pol}_{R}, 5^{\prime}$ AATGCCATGAGAAATGCTTCCAATT3'; $\mathrm{Nef}_{F}, 5^{\prime}$ CTCTCTGCGACCCTACAGAGG3'; Nef $_{R}, 5^{\prime}$ GCATTTCGCTCTGTATTCAGTC3'. These fragments were then cloned into pCR2.1 (Invitrogen) and sequenced. A minimum of five clones of each gene were sequenced for each time point for each animal to determine the consensus sequence. Any differences in sequence were resolved by repeated cloning and sequencing. In animals in which virus could not be isolated by coculture, but viral loads were detected by NASBA, the viral epitopes were sequenced by utilizing the viral RNA extracted from plasma for the NASBA assay. In these samples, a calibrator RNA was added for the NASBA assay; the calibrator consisted of a mismatched Gag sequence and that precluded our cloning of Gag epitopes for these time points.

2.6. Statistical Analysis. All statistical analyses were performed with GraphPad Prism (Windows version 4.03, GraphPad Software, San Diego, CA). Data was first tested for normality using the Kolmogorov-Smirnov (KS) test, with the $P$-value determined by the Dallal and Wilkinson test. The acute phase data sets of both the vaccinated and unvaccinated groups failed the normality test $(P=.0253$ and $P<.0001$, resp.). Data were then transformed to $\log$ scale to account for the variability of animal modelling and to improve further statistical analyses by normalizing the data. Subsequent analyses are detailed in the results and included, Mann-Whitney comparison of mean responses, as well as Pearson's correlation to determine significant correlations of the CTL response over time.

\section{Results}

3.1. Outcome of SIV Challenge and Expression of Protective Alleles. Using the tether system to study the acute phase after viral challenge, we recently demonstrated that all the macaques inoculated with the live-attenuated SIV vaccine were protected from pathogenic SIV infection [32]. As Table 1 shows however, while three $(16037,16041$, and 16044) of four animals controlled SIV infection very efficiently and remained SP or LTNP, one vaccinated animal (16040, Мати $A^{*} 01^{+}$) progressed to simian AIDS (SAIDS) at 118 weeks postchallenge. On the other hand, and as expected, unvaccinated animals became infected with SIV and progressed to disease at 12 (11896), 16 (12900), and 52 (12416) weeks postchallenge (WPC); one naïve animal, however, spontaneously controlled infection and remained as LTNP (14757). Three of the four animals that controlled infection had the protective Mamu $A^{*} 01$ allele $(16037,16041$, and 14757); the naive LNTP animal (14757) also had the Mamu $B^{*} 08$ allele.

3.2. Epitope Emergence in Slow- and Long- Term Nonprogressors. Table 1 also includes a summary of the epitopes that were recognized by CTL responses (as measured by IFN- $\gamma$ ELISPOT responses) that significantly changed over the course of infection. The epitopes that triggered IFN- $\gamma$ ELISPOT responses were also shown to induce degranulation (surface expression of CD107a) of $\mathrm{CD}^{+} \mathrm{T}$ cells and to induce killing of autologous lymphoblastoid B cell lines (data not shown). The SP/LTNP group included two vaccinated animals with the Mamu $A^{*} 01$ allele, 16037 and 16041, and one naive animal with the Mamu $A^{*} 01$ and $B^{*} 08$ alleles, 14757. All animals in the Мати $A^{*} 01$ positive subgroup showed a statistically significant positive correlation in the CTL response to the previously described Mamu $A^{*} 01$ restricted immunodominant Gag CTPYDINQM epitope [35]. None of the viral loads in these animals exceeded 500 RNA genome copies per $100 \mu \mathrm{l}$ of plasma in the 4 years of this study. In addition, unvaccinated animal 14757 had significant increases in CTL responses to the recently reported Mamu $B^{*} 08$ restricted Nef RRLTARGLL epitope and to an unreported putative Gag TAPSSGRGGNY epitope late in infection.

The fourth animal in the SP/LTNP group was the vaccinated SP animal 16044, who lacked Mamu $A^{*} 01$, Mamu $B^{*} 08$, and Mamu $B^{*} 17$ alleles as confirmed by cloning and sequence-specific PCR screening, and showed a significant negative trend in a previously unreported putative Vif epitope YFPCFTAGEVR. The establishment of an early strong CTL response to the Vif YFPCFTAGEVR epitope, 


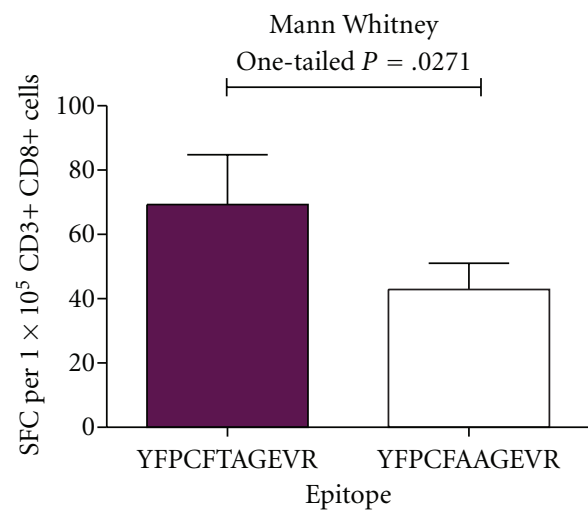

(a)

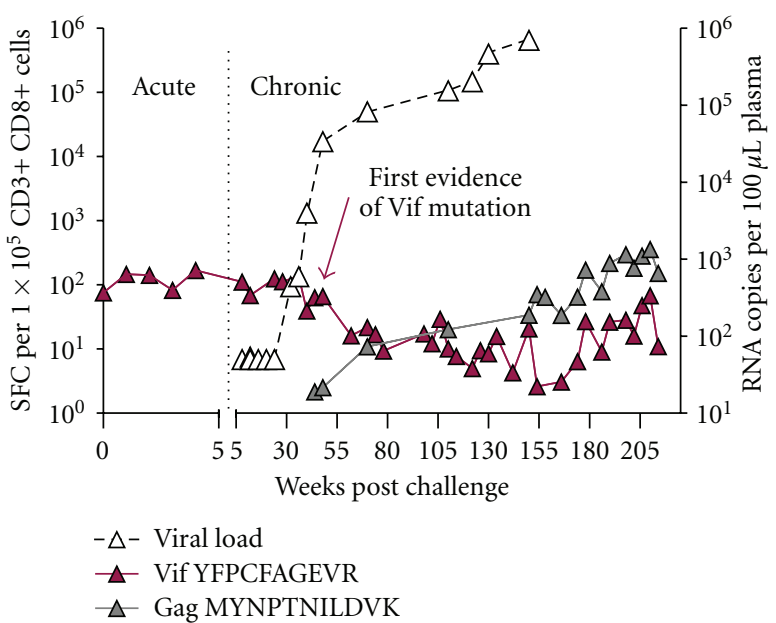

(b)

Figure 1: Viral Evolution in an Animal with no Known Protective Mamu Alleles. (a) Comparison of Vif-specific IFN- $\gamma$ ELISPOT values for the vaccinated $M a m u-A^{*} 01^{-},-B^{*} 08^{-},-B^{*} 17^{-}$SP animal 16044 before and after an observed Vif YFPCFTAGEVR to YFPCFAAGEVR mutation. Statistical analysis was performed using the Mann Whitney test. (b) NASBA viral loads and ELISPOT results for acute (Vif) and chronic (Gag) epitopes were compared to determine the ability of CTLs targeting either the Gag or Vif epitope to control viral replication.
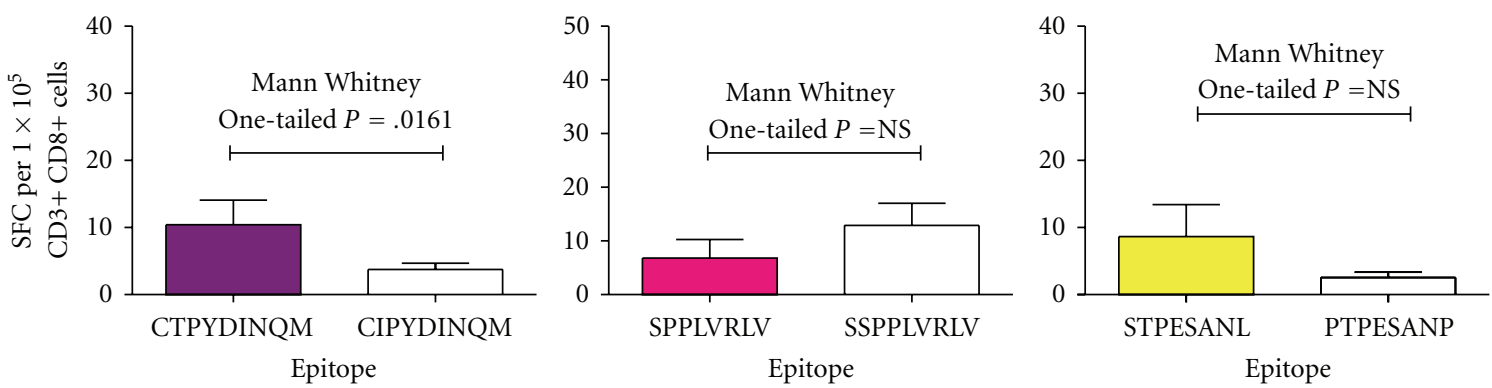

(a)

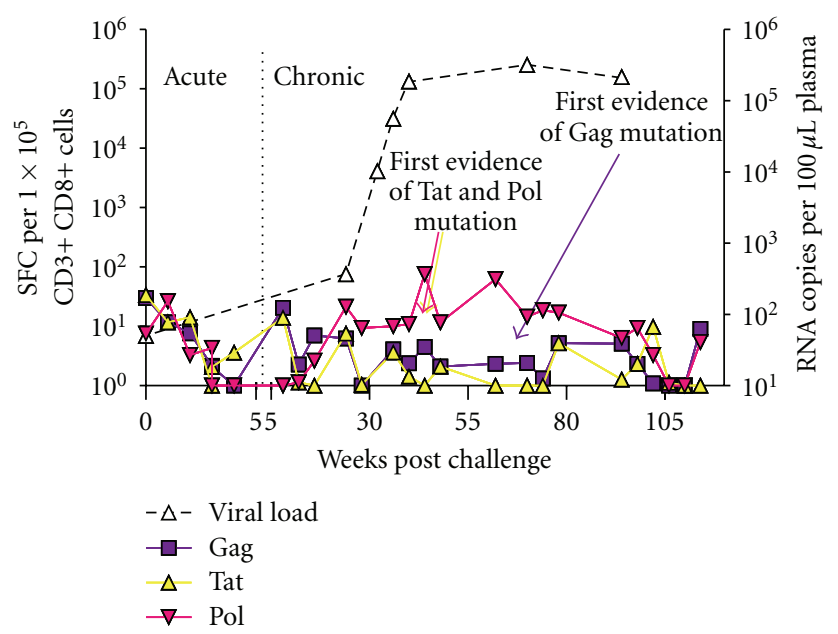

(b)

Figure 2: Viral Evolution in an Animal with Known Protective Mamu Alleles. (a) Comparison of IFN- $\gamma$ ELISPOT values for the vaccinated, Mamu $A^{*} 01^{+} \mathrm{NP}$ animal 16040 before or after the Mamu $A^{*} 01$-restricted Gag CTPYDINQM to CIPYDINQM, Tat STPESANL to PTPESANP, and Pol STPPLVRLV to SSPPLVRLV mutations. Statistical analysis was performed using the Mann Whitney test. (b) NASBA viral loads and IFN- $\gamma$ ELISPOT results for all three epitopes were plotted to determine the effects of mutations on the control of viral replication. 
TABLE 1: MHC class I allele and CTL Epitope Summary.

\begin{tabular}{|c|c|c|c|c|c|c|}
\hline $\begin{array}{l}\text { Animal } \\
\text { Number }\end{array}$ & $\begin{array}{l}\text { Vaccination, } \\
\text { Disease } \\
\text { Progression }\end{array}$ & Mamu alleles & CTL Epitope & $\begin{array}{l}\text { Significant } \\
\text { Trend in CTL } \\
\text { Response }\end{array}$ & $\begin{array}{l}\text { Emergence } \\
\text { of viral } \\
\text { mutants }\end{array}$ & $\begin{array}{l}\text { Restricting } \\
\text { Allele for CTL } \\
\text { Epitope }\end{array}$ \\
\hline \multirow[t]{2}{*}{16037} & LAV, LTNP & $\begin{array}{l}\mathbf{A}^{*} 01^{[1]}, A^{*} 02 \\
A^{*} 04\end{array}$ & Gag CTPYDINQM* & Increasing & No & $A^{*} 01$ \\
\hline & & $\begin{array}{l}\mathrm{B}^{*} 07 \\
\mathrm{~B}^{*} 09 \mathrm{~V}^{[2]}\end{array}$ & Tat STPESANL & Increasing & No & $A^{*} 01$ \\
\hline \multirow[t]{2}{*}{16041} & LAV, LTNP & $\begin{array}{l}\mathrm{A}^{*} 01, \mathrm{~A}^{*} 23, \mathrm{~B} \\
\text { alleles not }\end{array}$ & Gag CTPYDINQM* & Increasing & Unknown & $\mathrm{A}^{*} 01$ \\
\hline & & determined & Tat STPESANL & Decreasing & Unknown & $\mathrm{A}^{*} 01$ \\
\hline \multirow[t]{4}{*}{14757} & None, LTNP & $\begin{array}{l}\mathrm{A}^{*} 01, \mathrm{~A}^{*} 04, \\
\mathrm{~A}^{*} 23 \mathrm{~V}\end{array}$ & Gag CTPYDINQM* & Increasing & No & $\mathrm{A}^{*} 01$ \\
\hline & & $\mathrm{B}^{*} 07, \mathrm{~B}^{*} \mathbf{0 8}$ & Nef RRLTARGLL & Increasing & No & $\mathrm{B}^{*} 08$ \\
\hline & & & Tat STPESANL & Increasing & Yes & $\mathrm{A}^{*} 01$ \\
\hline & & & Gag TAPSSGRGGNY & Increasing & No & Unknown \\
\hline \multirow[t]{3}{*}{16044} & LAV, SP & $\begin{array}{l}\mathrm{A}^{*} 04 \mathrm{~V}, \mathrm{~A}^{*} 23 \\
\mathrm{~B}^{*} 3002 \mathrm{~V}\end{array}$ & Vif YFPCFTAGEV $\mathrm{R}^{* \mathrm{P}}$ & Decreasing & Yes & Unknown \\
\hline & & $\begin{array}{l}\mathrm{B}^{*} 09 \mathrm{~V}, \mathrm{~B}^{*} 21 \\
\mathrm{~B}^{*} 49\end{array}$ & Gag EQIQWMYRQQ $^{P}$ & Decreasing & No & Unknown \\
\hline & & & Gag MYNPTNILDVK ${ }^{\mathrm{P}}$ & Increasing & No & Unknown \\
\hline 16040 & $\begin{array}{l}\text { LAV, NP } \\
(118 \mathrm{~W})\end{array}$ & $\begin{array}{l}\mathrm{A}^{*} \mathbf{0 1}, \mathrm{A}^{*} 23 \mathrm{~V} \\
\mathrm{~B}^{*} 63 \mathrm{~N}\end{array}$ & Gag CTPYDINQM* & Decreasing & Yes & $A^{*} 01$ \\
\hline 12416 & $\begin{array}{l}\text { None, NP } \\
(52 \mathrm{~W})\end{array}$ & $\begin{array}{l}\mathrm{A}^{*} 04, \\
\mathrm{~A}^{*} 25 \mathrm{~N}^{[3]}, \\
\mathrm{B}^{*} 67\end{array}$ & Nef RTMSYKLAIDM ${ }^{\mathrm{P}}$ & Increasing & Yes & Unknown \\
\hline 11896 & $\begin{array}{l}\text { None, RP } \\
(12 \mathrm{~W})\end{array}$ & ND & ND & ND & ND & ND \\
\hline 12900 & $\begin{array}{c}\text { None, RP } \\
(16 \mathrm{~W})\end{array}$ & $\begin{array}{l}\mathrm{A}^{*} 02, \mathrm{~A}^{*} 02 \mathrm{~V} \\
\mathrm{~A}^{*} 04, \mathrm{~B}^{*} 05 \mathrm{~V} \\
\mathrm{~B}^{*} 07\end{array}$ & ND & ND & ND & ND \\
\hline
\end{tabular}

LAV: live-attenuated vaccine, LTNP: long-term non progressor, SP: slow prog., NP: normal prog., RP: rapid prog., (time of euthanasia in weeks post-challenge).

${ }^{[1]}$ Bolded alleles have been reported as protective.

${ }^{[2]}$ Variant (less than 5 amino acid changes in $\alpha 1$ and $\alpha 2$ domains from published sequence of allele listed).

${ }^{[3]}$ Novel (more than 5 amino acid differences in $\alpha 1$ and $\alpha 2$ domains from published sequence of allele listed).

${ }^{\mathrm{P}}$ Putative Epitope, ${ }^{*}$ Immunodominant Epitope.

coupled with initial control of SIV replication, implied that this might be an epitope with protective characteristics. This epitope is located in a functionally important area of the Vif protein and is highly conserved across SIV and HIV strains. The Vif YFPCFTAGEVR epitope showed a threonine (T) to alanine (A) mutation within the putative epitope first noted at 48 weeks post-challenge with no apparent increase in viral replication prior to that time. To determine what effect the CTL response was having in protecting this animal from progressing to disease, we performed additional ELISPOT pool studies on fresh and frozen PBMC samples. As expected, CTL responses to the wild-type peptide were significantly lower at timepoints at which the circulating virus had mutated ( $P=.0271$, one-tailed Mann Whitney test, Figure 1(a)), which is consistent with previous studies on escape mutations and implies that the CTL population specific for the wild-type epitope was declining, presumably because the virus in the animal no longer had the wild-type epitope. Pool studies on archival PBMC samples identified additional epitopes in the Gag protein that were being targeted by CTLs. The strongest ELISPOT response was seen with the emergence of the putative Gag MYNPTNILDVK epitope $(<45$ WPC versus $>45$ WPC, $P<.0022$, onetailed Mann Whitney test) that occurred after 45 weeks post-challenge, which was concurrent with a significant loss of response to the Vif YFPCFTAGEVR epitope ( $<45$ WPC versus $>45$ WPC, $P<.0001$, one-tailed Mann Whitney test). Coincidentally, viral loads steadily increased from this time point onwards, despite strong CTL responses to Gag MYNPTNILDVK (Figure 1(b)). Whether the loss of control over viral replication by CTLs targeting these epitopes was due to viral escape, an intrinsic deficit of effector functions, or some other silencing mechanism such as increased PD-1 expression, was not determined.

3.3. Epitope Emergence in Normal and Rapid Progressors. Not all animals harbouring the protective $M a m u A^{*} 01$ allele were able to control SIV infection. Sequencing of the viral epitopes in the Mamu $A^{*} 01^{+} 16040$ animal that progressed to SAIDS at 118 weeks post-challenge indicated that a threonine $(T)$ 


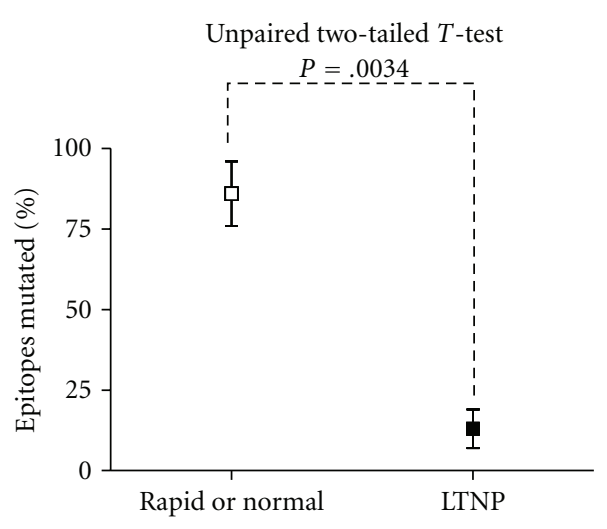

(a)

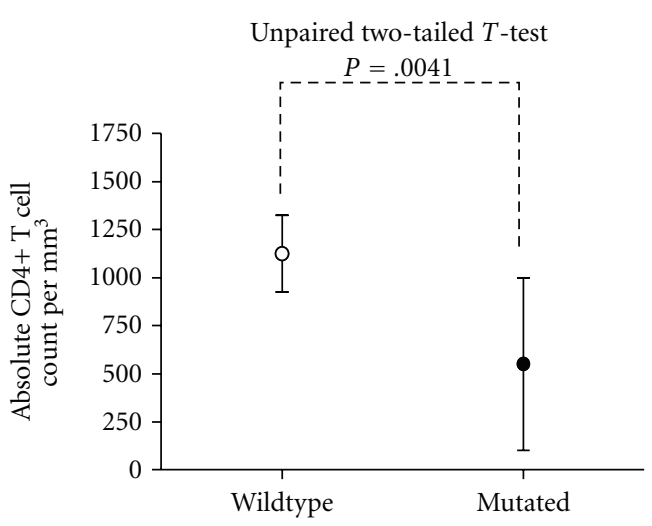

(b)

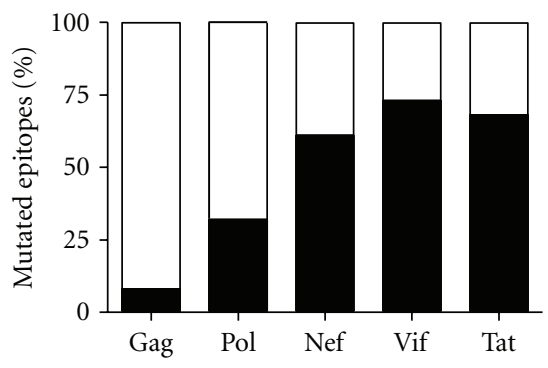

Mutated epitopes Wildtype epitopes

(c)

FIgure 3: Viral Evolution and Disease Progression. (a) Sequencing was performed for all recognized CTL epitopes in the ELISPOT assay (3-5 clones per epitope and 2-3 epitopes per animal) for each animal ( 6 animals) at all time points that the animal was virus positive (2-5 per animal) ( $n=24-72$ clones per analysis). Accumulated mutations were noted and the percent of epitopes that showed mutations (regardless of the effect of the mutation on CTL responses) were compared between the NP/RP and SP/LTNP groups. (b) CD4 T cell absolute counts were measured at every time-point in which ELISPOT assays were performed and these counts were compared between animals carrying virus with wild-type epitopes or mutated epitopes. (c) Comparison of the number of accumulated mutations observed in CTL epitopes in viral proteins. Statistical analysis was performed using unpaired two-tail $t$-tests.

to isoleucine $(I)$ mutation was first observed in position 2 of the Gag CTPYDINQM epitope at 70 WPC. This is a well characterized escape mutation known to abrogate MHC binding [26] and was associated with a significant decrease in CTL response (Figure 2(a)). Other known escape mutations were first detected at 48 WPC in position 1 and 8 of the Tat STPESANL epitope (serine to proline, and leucine to proline) and these have also been reported to abrogate MHC binding [26]. This is consistent with declining numbers of CTLs specific for the wild-type epitope, which would be expected in animals no longer carrying virus with wildtype sequence. Interestingly, a threonine $(T)$ to serine $(S)$ mutation was observed in position 2 of the Mamu $A^{*} 01 \mathrm{Pol}$ STPPLVRLV epitope prior to 48WPC, but was not associated with a decrease in CTL response (Figure 2(a)). Analysis of viral loads (Figure 2(b)) illustrated that the Pol STPPLVRLV epitope was not protective as the viral load increased despite a CTL response to this epitope.

The unvaccinated normal progressor macaque 12416 (lacking known protective alleles) showed an initial ELISPOT response to a Gag peptide pool that declined before individual epitopes could be deconvoluted. This animal had significant ELISPOT response increase over time to a putative Nef epitope RTMSYKLAIDM. Despite the increased response to this epitope, the viral load at 54 WPC was 420,270 RNA genome copies per $100 \mu \mathrm{L}$ of plasma (data not shown). Neither of the rapid progressors $(12900,11896)$ showed any detectable ELISPOT response.

3.4. Mutations in Relation to Disease Progression. We counted the number of mutations that we identified for each animal throughout the course of this study and its relation to disease progression. We found significantly higher percentages of CTL epitope mutations in the normal or rapid progressing animals (Figure 3(a)) as compared to SP/LTNP animals. Animals that carried viruses with wild-type CTL epitopes maintained significantly higher CD4 $+\mathrm{T}$ cells counts than animals with virus that has mutated at CTL epitopes (Figure 3(b)). To determine patterns in the emergence of CTL epitope mutations, we also compared the percentage of mutated CTL epitopes found on each viral protein for all time points and observed that accessory proteins appeared to 
mutate at a higher frequency than the structural proteins in this sample (Figure 3(c)).

\section{Discussion}

Data generated from our studies during the chronic phase of SIV infection adds to the understanding of viral evolution in the context of CTL selection by analyzing epitope evolution over 4 years in slow- and long-term nonprogressors, as well as normal and rapid progressors. The importance of this study lies in the observation, albeit in a small group of animals, that the loss of immunodominant CTL responses established in acute infection may lead to disease progression regardless of the expression of known protective class I MHC alleles. In addition, this increase in viral replication occurred despite late-emerging CTL responses of similar magnitude to the one seen for dominant epitopes. Previous studies using animals with Mamu $A^{*} 01$ and/or Mamu $A^{*} 02$ alleles have demonstrated that the evolution of CTL responses to subdominant epitopes can arise after loss of Мати $A^{*} 01$ immunodominant responses [36], but a loss of immunodominant responses is followed by increased viral replication, even if normal CTL responses to subdominant epitopes (Pol) are present [20,37]; our data from the Mamu $A^{*} 01^{+}$animal 16040 (Figure 2) agrees with this observation. In the same line of evidence, and outside the context of the Mamu $A^{*} 01$ allele expression, the loss of the initial immunodominant Vif CTL epitope in animal 16044, resulted in increased viral replication and disease progression despite strong surge of subdominant CTLs (Figure 1). These findings in the SIV system are also in agreement with results from a case report of a closely studied HIV-infected individual [38] and a recent study in a large cohort of acutely HIV-infected individuals that were followed into the chronic phase of the infection, and that showed that the preservation of the initial CD8+ T-cell immunodominance patterns from the acute into the chronic phase of infection was significantly associated with slower CD4+ T-cell decline and subsequent control of viremia [39]. These results appear to be in conflict with a previous study that found that subdominant CTL responses may have been associated with in vivo HIV-1 viral control [40]; however, this latter study utilized few time-points from chronic HIV-infected patients, whereas the study of Streeck et al. [39], like our study, followed the CTL evolution of acutely HIV-1-infected subjects that progressed to chronicity. Similarly to the results of Frahm et al. [40], Streeck et al. did not find an association between immunodominant CTLs and viral control when samples from the chronic phase were evaluated; however, like we report here, the positive correlation was observed when the immunodominant CTLs that aroused in the acute phase of infection were still maintained in the chronic phase [39].

Our studies also identified a novel SIV epitope that elicited strong CTL responses outside of known protective class I MHC allele expression. The putative Vif YFPCFTAGEVR epitope was the target of a protective CTL response for a slow progressing animal (16044) that did not express any known protective allele. This peptide sequence is highly conserved in various HIV and SIV strains including SIVsmm, SIVmac, HIV2a, and HIV2b; mutagenesis of the Vif protein has identified the same region of the protein as functionally important, with the mutations in the tyrosine $(\mathrm{Y})$, phenylalanine $(\mathrm{F})$, or cysteine $(\mathrm{C})$ residues reducing activity of the protein to 1 to $15 \%$ of wild type [41]. This observation is in agreement with recent studies in HIV-1infected individuals that suggest that control of infection may be associated with acute-phase CD8 responses capable of selecting for viral escape mutations in highly conserved regions of the virus [42]. In addition, we demonstrate that slow- and long-term nonprogressors acquire fewer mutations in CTL epitopes than normal or rapid progressors and a higher $\mathrm{CD} 4+\mathrm{T}$ cell count was associated with CTL responses to wild-type epitopes (Figure 3). This is similar to what has been observed for a large cohort of HIV infected individuals [43] and implies that the protection achieved with certain alleles can be attributed to a failure of the virus to accumulate escape mutations.

All these observations combined suggest that natural containment of AIDS virus replication may be related to a combination of immunodominance and viral escape from $\mathrm{CD}^{+} \mathrm{T}$-cell responses; that is, those individuals who generate acute dominant CTL that target constrained viral epitopes may have a better chance to control the infection.

\section{Conclusions}

This study contributes to the body of knowledge regarding CTL immune responses in SIV- infected rhesus macaques and increases our understanding of viral evolution in the context of SIV infection. Although the sample group is small, our observations are detailed and consistent with previous reports and contribute to the validation of the SIV/rhesus macaque model of AIDS. In addition, our identification of novel epitopes provides preliminary data justifying the continued analysis of CTL populations in these animals to better understand CTL function. Our data also add to the understanding of the associations between CTL epitope mutation and disease progression and CD4+ T cell counts and imply that accessory proteins accrue mutations more frequently. Lastly, we show that a loss of immunodominant immune responses to conserved viral epitopes can lead to disease progression despite maintained or emerging CTL responses outside the context of known protective allele expression, which has implications for the rational selection of antigens for HIV vaccine design.

\section{Authors' Contributions}

M. S. Keckler performed the majority of the assays described here and drafted the manuscript. V. L. Hodara trained M. S. Keckler and assisted in all other assays. L. M. Parodi performed the cell cultures, ELISAs and viral isolations. L. D. Giavedoni conceived of and designed the study, mentored M. S. Keckler, and edited the paper. All authors read and approved the final paper. 


\section{Acknowledgments}

This work was supported by the Public Health Service Grants P51 RR013986 (the National Center for Research Resources), R03 AI55443 (the National Institute for Allergy and Infectious Diseases), and R21 AI55369 (the National Institute for Allergy and Infectious Diseases). This investigation was conducted in a facility constructed with support from Research Facilities Improvement Program Grant no. C06 RR12087 from the National Center for Research Resources, the National Institutes of Health. The authors thank the personnel from Veterinary and Research Resources of the Southwest National Primate Research Center and Ms. April Hopstetter for editing this paper.

\section{References}

[1] V. Appay, D. C. Douek, and D. A. Price, "CD8 ${ }^{+}$T cell efficacy in vaccination and disease," Nature Medicine, vol. 14, no. 6, pp. 623-628, 2008.

[2] L. E. Valentine and D. I. Watkins, "Relevance of studying T cell responses in SIV-infected rhesus macaques," Trends in Microbiology, vol. 16, no. 12, pp. 605-611, 2008.

[3] D. T. Evans, P. Jing, T. M. Allen, et al., "Definition of five new simian immunodeficiency virus cytotoxic t-lymphocyte epitopes and their restricting major histocompatibility complex class I molecules: evidence for an influence on disease progression," Journal of Virology, vol. 74, no. 16, pp. 74007410, 2000.

[4] R. Pal, D. Venzon, N. L. Letvin, et al., "ALVAC-SIV-gag-polenv-based vaccination and macaque major histocompatibility complex class I $\left(\mathrm{A}^{*} 01\right)$ delay simian immunodeficiency virus SIVmac-induced immunodeficiency," Journal of Virology, vol. 76, no. 1, pp. 292-302, 2002.

[5] G. M. A. Gillespie, R. Kaul, T. Dong, et al., "Cross-reactive cytotoxic T lymphocytes against a HIV-1 p24 epitope in slow progressors with $\mathrm{B}^{*} 57$," AIDS, vol. 16, no. 7, pp. 961-972, 2002.

[6] M. J. Kuroda, J. E. Schmitz, D. H. Barouch, et al., "Analysis of gag-specific cytotoxic T lymphocytes in simian immunodeficiency virus-infected rhesus monkeys by cell staining with a tetrameric major histocompatibility complex class I-peptide complex," Journal of Experimental Medicine, vol. 187, no. 9, pp. 1373-1381, 1998.

[7] W. R. Morton, M. B. Agy, S. V. Capuano, and R. F. Grant, "Specific pathogen-free macaques: definition, history, and current production," ILAR Journal, vol. 49, no. 2, pp. 137-144, 2008.

[8] L. J. Yant, T. C. Friedrich, R. C. Johnson, et al., "The highfrequency major histocompatibility complex class I allele Mamu- ${ }^{*} 17$ is associated with control of simian immunodeficiency virus SIVmac239 replication," Journal of Virology, vol. 80, no. 10, pp. 5074-5077, 2006.

[9] J. A. Wojcechowskyj, L. J. Yant, R. W. Wiseman, S. L. O'Connor, and D. H. O'Connor, "Control of simian immunodeficiency virus SIVmac239 is not predicted by inheritance of Mamu-B*17-containing haplotypes," Journal of Virology, vol. 81, pp. 406-410, 2007.

[10] J. T. Loffredo, J. Maxwell, Y. Qi, et al., "Mamu-B*08positive macaques control simian immunodeficiency virus replication," Journal of Virology, vol. 81, pp. 8827-8832, 2007.
[11] X. G. Yu, M. M. Addo, E. S. Rosenberg, et al., "Consistent patterns in the development and immunodominance of human immunodeficiency virus type 1 (HIV-1)-specific CD8 ${ }^{+}$ T-cell responses following acute HIV-1 infection," Journal of Virology, vol. 76, no. 17, pp. 8690-8701, 2002.

[12] R. Draenert, T. M. Allen, Y. Liu, et al., "Constraints on HIV1 evolution and immunodominance revealed in monozygotic adult twins infected with the same virus," Journal of Experimental Medicine, vol. 203, no. 3, pp. 529-539, 2006.

[13] S. Tenzer, E. Wee, A. Burgevin, et al., "Antigen processing influences HIV-specific cytotoxic T lymphocyte immunodominance," Nature Immunology, vol. 10, no. 6, pp. 636-646, 2009.

[14] C. L. Day, A. K. Shea, M. A. Altfeld, et al., "Relative dominance of epitope-specific cytotoxic T-lymphocyte responses in human immunodeficiency virus type 1-infected persons with shared HLA alleles," Journal of Virology, vol. 75, no. 14, pp. 6279-6291, 2001.

[15] I. Messaoudi, J. A. Guevara Patiño, R. Dyall, J. LeMaoult, and J. Nikolich-Zugich, "Direct link between mhc polymorphism, T cell avidity, and diversity in immune defense," Science, vol. 298, no. 5599, pp. 1797-1800, 2002.

[16] A. Hasegawa, C. Moriya, H. Liu, et al., "Analysis of $\operatorname{TCR} \alpha \beta$ combinations used by simian immunodeficiency virusspecific $\mathrm{CD}^{+} \mathrm{T}$ cells in rhesus monkeys: implications for CTL immunodominance," Journal of Immunology, vol. 178, no. 6, pp. 3409-3417, 2007.

[17] K. C. Pang, M. T. Sanders, J. J. Monaco, P. C. Doherty, S. J. Turner, and W. Chen, "Immunoproteasome subunit deficiencies impact differentially on two immunodominant influenza virus-specific $\mathrm{CD}^{+} \mathrm{T}$ cell responses," Journal of Immunology, vol. 177, no. 11, pp. 7680-7688, 2006.

[18] M. F. Kotturi, I. Scott, T. Wolfe, et al., "Naive precursor frequencies and MHC binding rather than the degree of epitope diversity shape CD8 ${ }^{+} \mathrm{T}$ cell immunodominance," Journal of Immunology, vol. 181, no. 3, pp. 2124-2133, 2008.

[19] C. Brander, N. Frahm, and B. D. Walker, "The challenges of host and viral diversity in HIV vaccine design," Current Opinion in Immunology, vol. 18, no. 4, pp. 430-437, 2006.

[20] D. O'Connor, T. Friedrich, A. Hughes, T. M. Allen, and D. Watkins, "Understanding cytotoxic T-lymphocyte escape during simian immunodeficiency virus infection," Immunological Reviews, vol. 183, pp. 115-126, 2001.

[21] D. H. O'Connor, T. M. Allen, T. U. Vogel, et al., "Acute phase cytotoxic T lymphocyte escape is a hallmark of simian immunodeficiency virus infection," Nature Medicine, vol. 8, no. 5, pp. 493-499, 2002.

[22] D. A. Price, S. M. West, M. R. Betts, et al., "T cell receptor recognition motifs govern immune escape patterns in acute SIV infection," Immunity, vol. 21, no. 6, pp. 793-803, 2004.

[23] J. T. Loffredo, T. C. Friedrich, E. J. Leon, et al., "CD8 ${ }^{+}$T cells from SIV elite controller macaques recognize Mamu-B*08bound epitopes and select for widespread viral variation," PLoS One, vol. 2, article e1152, 2007.

[24] A. B. McDermott, D. H. O'Connor, S. Fuenger, et al., "Cytotoxic T-lymphocyte escape does not always explain the transient control of simian immunodeficiency virus SIVmac239 viremia in adenovirus-boosted and DNA-primed Mamu-A*01-positive rhesus macaques," Journal of Virology, vol. 79, pp. 15556-15566, 2005.

[25] N. J. Maness, L. J. Yant, and C. Chung, "Comprehensive immunological evaluation reveals surprisingly few differences between elite controller and progressor Mamu-B* 17 -positive 
Simian immunodeficiency virus-infected rhesus macaques," Journal of Virology, vol. 82, pp. 5245-5254, 2008.

[26] Z. W. Chen, A. Craiu, L. Shen, et al., "Simian immunodeficiency virus evades a dominant epitope-specific cytotoxic $\mathrm{T}$ lymphocyte response through a mutation resulting in the accelerated dissociation of viral peptide and MHC class I," Journal of Immunology, vol. 164, no. 12, pp. 6474-6479, 2000.

[27] F. W. Peyerl, D. H. Barouch, W. W. Yeh, et al., "Simian-human immunodeficiency virus escape from cytotoxic T-lymphocyte recognition at a structurally constrained epitope," Journal of Virology, vol. 77, no. 23, pp. 12572-12578, 2003.

[28] T. C. Friedrich, E. J. Dodds, L. J. Yant, et al., "Reversion of CTL escape-variant immunodeficiency viruses in vivo," Nature Medicine, vol. 10, no. 3, pp. 275-281, 2004.

[29] T. C. Friedrich, C. A. Frye, L. J. Yant, et al., "Extraepitopic compensatory substitutions partially restore fitness to simian immunodeficiency virus variants that escape from an immunodominant cytotoxic-T-lymphocyte response," Journal of Virology, vol. 78, no. 5, pp. 2581-2585, 2004.

[30] M. Kobayashi, H. Igarashi, A. Takeda, M. Kato, and T. Matano, "Reversion in vivo after inoculation of a molecular proviral DNA clone of simian immunodeficiency virus with a cytotoxic-T-lymphocyte escape mutation," Journal of Virology, vol. 79, no. 17, pp. 11529-11532, 2005.

[31] V. L. Hodara, M. Velasquillo, L. M. Parodi, and L. D. Giavedoni, "Expression of CD154 by a simian immunodeficiency virus vector induces only transitory changes in rhesus macaques," Journal of Virology, vol. 79, no. 8, pp. 4679-4690, 2005.

[32] M. S. Keckler, V. L. Hodara, L. M. Parodi, and L. D. Giavedoni, "Novel application of nonhuman primate tethering system for evaluation of acute phase SIVmac251 infection in rhesus macaques (macaca mulatta)," Viral Immunology, vol. 20, pp. 623-634, 2007.

[33] D. R. Johnson, "Differential expression of human major histocompatibility class I loci: HLA-A,-B, and -C," Human Immunology, vol. 61, no. 4, pp. 389-396, 2000.

[34] M. Kaizu, G. J. Borchardt, C. E. Glidden, et al., "Molecular typing of major histocompatibility complex class I alleles in the Indian rhesus macaque which restrict SIV $\mathrm{CD}^{+} \mathrm{T}$ cell epitopes," Immunogenetics, vol. 59, no. 9, pp. 693-703, 2007.

[35] Z. W. Chen, H. Yamamoto, D. I. Watkins, G. Levinson, and N. L. Letvin, "Predominant use of a T-cell receptor V $\beta$ gene family in simian immunodeficiency virus gag-specific cytotoxic T lymphocytes in a rhesus monkey," Journal of Virology, vol. 66, no. 6, pp. 3913-3917, 1992.

[36] M. Newberg, M. Kuroda, and W. Charini, "A simian immunodeficiency virus nef Peptide is a dominant cytotoxic T lymphocyte epitope in Indian-origin rhesus monkeys expressing the common MHC class I allele mamu- $a^{*} 02$," Virology, pp. 301-365, 2002.

[37] D. H. Barouch, J. Kunstman, M. J. Kuroda, et al., "Eventual AIDS vaccine failure in a rhesus monkey by viral escape from cytotoxic T lynphocytes," Nature, vol. 415, no. 6869, pp. 335339, 2002.

[38] A. Oxenius, D. A. Price, A. Trkola, et al., "Loss of viral control in early HIV-1 infection is temporally associated with sequential escape from $\mathrm{CD}^{+} \mathrm{T}$ cell responses and decrease in HIV-1-specific CD4+ and CD8 ${ }^{+}$T cell frequencies," Journal of Infectious Diseases, vol. 190, no. 4, pp. 713-721, 2004.

[39] H. Streeck, J. S. Jolin, Y. Qi, et al., "Human immunodeficiency virus type 1 -specific $\mathrm{CD}^{+} \mathrm{T}$-cell responses during primary infection are major determinants of the viral set point and loss of CD4+ T cells," Journal of Virology, vol. 83, no. 15, pp. 76417648, 2009.

[40] N. Frahm, P. Kiepiela, S. Adams, et al., "Control of human immunodeficiency virus replication by cytotoxic T lymphocytes targeting subdominant epitopes," Nature Immunology, vol. 7, no. 2, pp. 173-178, 2006.

[41] J. H. Simon, A. M. Sheehy, E. A. Carpenter, R. A. Fouchier, and M. H. Malim, "Mutational analysis of the human immunodeficiency virus type 1 Vif protein," Journal of Virology, vol. 73, no. 4, pp. 2675-2681, 1999.

[42] Y. E. Wang, B. Li, J. M. Carlson, et al., "Protective HLA class I alleles that restrict acute-phase $\mathrm{CD} 8^{+} \mathrm{T}$-cell responses are associated with viral escape mutations located in highly conserved regions of human immunodeficiency virus type 1 ," Journal of Virology, vol. 83, no. 4, pp. 1845-1855, 2009.

[43] Z. L. Brumme, C. J. Brumme, and D. Heckerman, "Evidence of differential HLA class I-mediated viral evolution in functional and accessory/regulatory genes of HIV-1," PLoS Pathogens, vol. 3, article e94, 2007. 


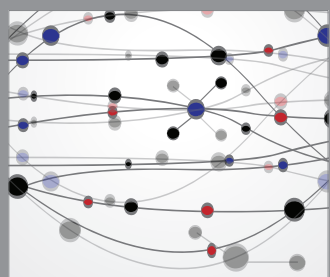

The Scientific World Journal
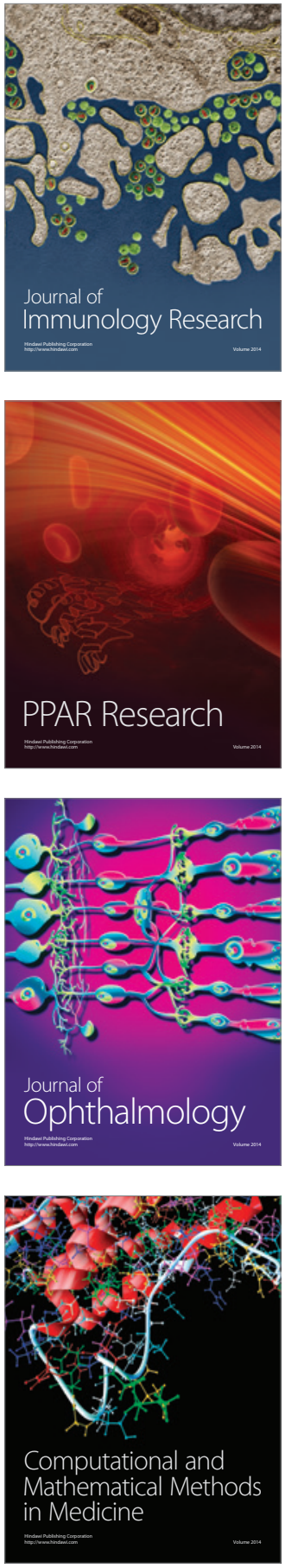

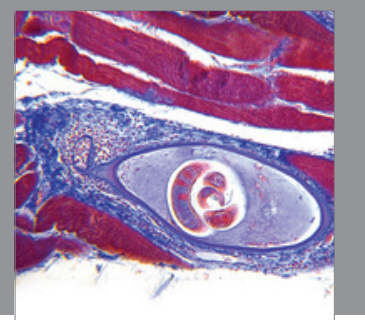

Gastroenterology

Research and Practice
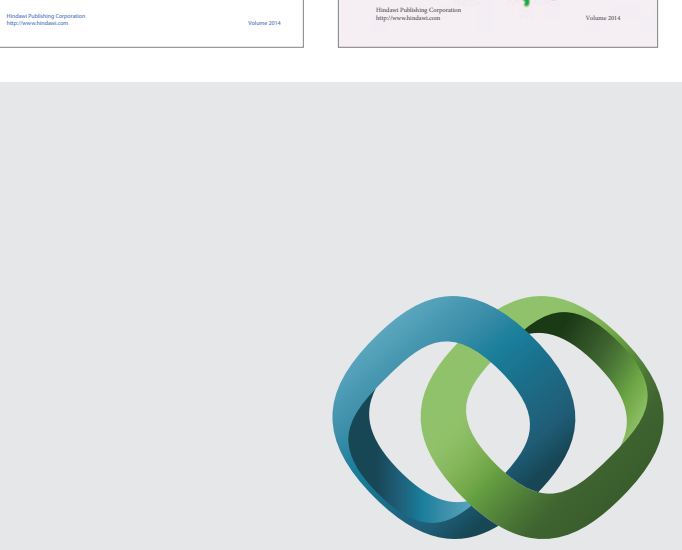

\section{Hindawi}

Submit your manuscripts at

http://www.hindawi.com
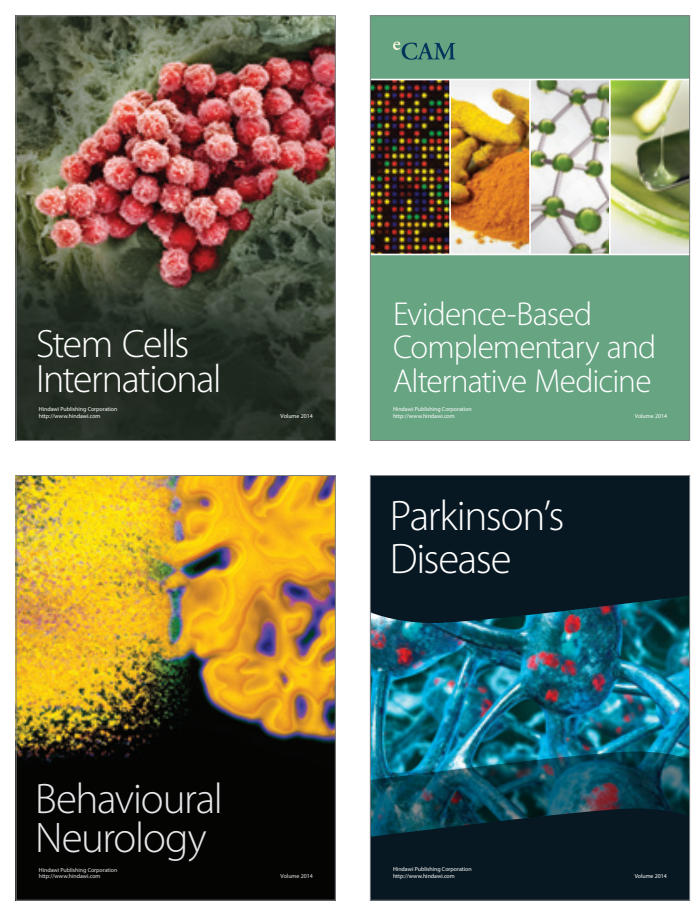

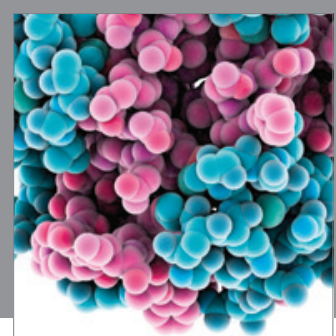

Journal of
Diabetes Research

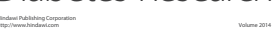

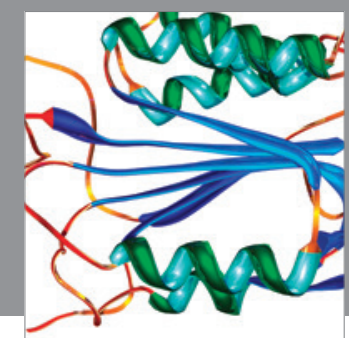

Disease Markers
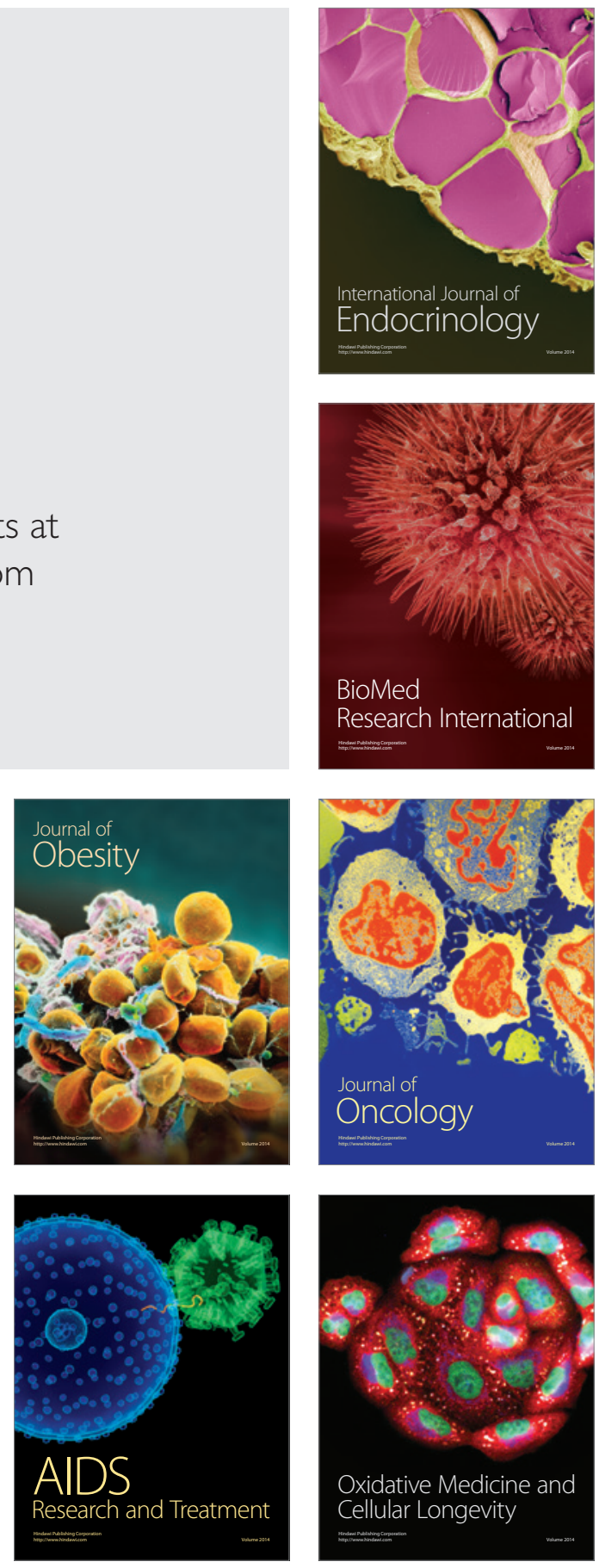